is naturaleza 


\section{DOSSIER CRÍTICO SOBRE EL LIBRO:}

\section{LOS SÓTANOS DEL UNIVERSO}

Volumen monográfico de

\section{NATURALEZA Y LIBERTAD}

Revista de estudios interdisciplinares

Número 3

Málaga, 2014

Esta revista es accesible on-line en el siguiente portal: http://grupo.us.es/naturalezayl 


\section{Naturaleza y Libertad}

Revista de estudios interdisciplinares

Número 3, 2014

ISSN: 2254-96682014

Directores: Juan Arana, Universidad de Sevilla; Juan José Padial, Universidad de Málaga; Francisco Rodríguez Valls, Universidad de Sevilla.

Secretaria: Avelina Cecilia Lafuente, Universidad de Sevilla.

Consejo de Redacción: José Luis González Quirós, Universidad Juan Carlos I, Madrid; Francisco Soler, Universität Dortmund / Universidad de Sevilla; Pedro Jesús Teruel. Universidad CEU Cardenal Herrera; Héctor Velázquez, Universidad Panamericana, México.

Adjunto a la redacción: Miguel Palomo, Universidad de Sevilla

Consejo Editorial: Mariano Álvarez, Real Academia de Ciencia Morales y Políticas; Allan Franklin, University of Colorado; Michael Heller, Universidad Pontificia de Cracovia; Manfred Stöcker, Universität Bremen; William Stoeger, University of Arizona.

Consejo Asesor: Rafael Andrés Alemañ Berenguer. Universidad de Alicante; Juan Ramón Álvarez, Universidad de León; Luciano Espinosa, Universidad de Salamanca; Miguel Espinoza, Université de Strasbourg; Juan A. García González, Universidad de Málaga; José Manuel Giménez Amaya, Universidad de Navarra; Karim Gherab Martín, Urbana University, Illinois; Martín López Corredoira, Instituto de Astrofísica de Canarias; Alfredo Marcos, Universidad de Valladolid; Javier Monserrat, Universidad Autónoma de Madrid; Leopoldo Prieto, Colegio Mayor San Pablo, Madrid; Ana Rioja, Universidad Complutense, Madrid. Madrid; José Luis González Recio, Universidad Complutense, Madrid; Javier Serrano, TEC Monterrey (México); Hugo Viciana, Université Paris I; Claudia Vanney, Universidad Austral, Buenos Aires; José Domingo Vilaplana, Huelva.

\section{Redacción y Secretaría:}

Naturaleza y Libertad. Revista de estudios interdisciplinares. Departamento de Filosofía y Lógica. Calle Camilo José Cela s.n. E-41018 Sevilla.

Depósito Legal: MA2112-2012

용 954.55.77.57 Fax: 954.55.16.78. E-mail: jarana@us.es 
Naturaleza y Libertad. Revista de estudios interdisciplinares. Número 3, 2014. ISSN: 2254-9668

\section{ÍNDICE}

PRESENTACIÓN.

DOSSIER CRÍTICO SOBRE EL LIBRO LOS SÓTANOS DEL UNIVERSO

Juan Arana (U. de Sevilla), El problema de la determinación y el diálogo interdisciplinar......11

Miguel Acosta (U. CEU San Pablo, Madrid), La epistemología del riesgo y la

relación ciencia-filosofía. . .25

Carlos del Ama (Madrid), Reflexiones con ocasión del libro Los sótanos del universo ...........57

Luciano Espinosa (U. de Salamanca), De los sótanos al ático del universo ............................93

Gonzalo Génova (U. Carlos III, Madrid), Turing y el rostro del universo ..........................101

Julio A. Gonzalo (U. Autónoma de Madrid), De los sótanos a los confines del universo ....111

Juan Meléndez Sánchez (U. Carlos III, Madrid), De riesgos y metáforas

Andrés Muñoz Machado (Universidad Politécnica de Madrid), Las matemáticas en

Los sótanos del universo

Javier Sánchez Cañizares (U. de Navarra), Sótanos del universo, espejos del alma

Francisco José Soler Gil (U. de Sevilla), Una filosofía de la naturaleza contracorriente y antisistema.

Ignacio Sols (U. Complutense, Madrid), Recuperando la causa formal sin abandonar la causa eficiente. .151

Juan Arana (U. de Sevilla), Los subsótanos del universo. Materiales para un debate. 167 
Naturaleza y Libertad. Revista de estudios interdisciplinares. Número 3, 2014. ISSN: 2254-9668

\title{
TURING Y EL ROSTRO DEL UNIVERSO ${ }^{1}$ \\ Gonzalo Génova \\ Universidad Carlos III de Madrid
}

\begin{abstract}
Resumen: Al hilo del comentario se habla de cosas y personas tan (aparentemente) dispares como Atapuerca, el proceso de hominización, experimentos mentales con bolas de acero, Galileo, Descartes, Blade Runner, Turing y formularios electrónicos en Internet, con el fin de recordar algo que se olvida una y otra vez: Dios está más allá del conocimiento que proporciona el método científico-experimental.
\end{abstract}

Palabras clave: método científico-experimental, existencia de Dios, test de Turing.

Abstract: This comment talks about things and people as (seemingly) disparate as Atapuerca, the process of hominization, thought experiments with steel balls, Galileo, Descartes, Blade Runner, Turing and electronic forms on the Internet, in order to recall something that is forgotten over and over again: God is beyond the knowledge that is provided by the experimental scientific method.

Keywords: experimental-scientific method, existence of God, Turing's test.

Recibido: 13 de enero de 2013. Aprobado: 12 de diciembre de 2013.

Recientemente, coincidiendo en el tiempo con la lectura de Los sótanos del universo, de Juan Arana, asistí a una conferencia sobre la evolución humana impartida por el Prof. José María Bermúdez de Castro, codirector de los yacimientos de Atapuerca y director del Museo de la Evolución Humana de

1 Comentario al libro de Juan Arana, Los sótanos del universo. La determinación natural y sus mecanismos ocultos, Madrid, Biblioteca Nueva, 2012. VI Sesión de Trabajo del Seminario Permanente Bibliográfico AEDOS, 30 de noviembre de 2012. 
Burgos $^{2}$. Al llegar el turno de preguntas hubo varias intervenciones en la misma línea por parte del público asistente, que podríamos expresar así: ¿es posible asegurar que en el proceso de hominización-humanización haya habido un punto de inflexión totalmente singular, un salto inexplicable desde el punto de vista científico? Se adivinaba en la pregunta, repetida en formas distintas una y otra vez, el deseo de encontrar una especie de prueba cientifica de una intervención extraordinaria de un ser supranatural, es decir, una prueba de la existencia de Dios. Como era de esperar, el biólogo no entró al trapo, limitándose con gran elegancia a lo que podía responder desde su especialidad, la paleoantropología. En resumen, vino a decir que, si queremos, podemos considerar extraordinario todo el proceso, pero en todo caso con los conocimientos actuales no podemos señalar en él ningún momento especialmente singular que no pueda ser explicado científicamente.

A mi modo de ver, en el proceso de hominización-humanización puede que haya, o puede que no, un salto brusco. En todo caso, para la fe religiosa es irrelevante: el salto brusco no prueba mejor la intervención divina que el proceso gradual. Tanto en uno como en otro es posible contemplar la acción creadora de Dios (y es posible también ignorarla). La clave para llegar a contemplar a Dios en la naturaleza no está en el análisis de los fenómenos, como si unos fenómenos apuntaran a Dios y otros más bien en dirección

2 J. M. Bermúdez de Castro, Hominización y humanización. Conferencia pronunciada en Ser bumano y crisis, V Encuentro de la Escuela de Pensamiento de Silos, Abadía de Silos, 17 de noviembre de 2012 (http://www.escueladepensamientodesilos.com/eventos/ser-humano-ycrisis-existencial-y-economica.html). 
Naturaleza y Libertad. Revista de estudios interdisciplinares. Número 3, 2014. ISSN: 2254-9668

contraria. En otras palabras, la intervención de Dios en la Historia no puede verificarse empiricamente.

Me gustaría ilustrar esta tesis con un experimento mental de corte galileano, que espero sirva de conveniente analogía al proceso evolutivo comentado. A Galileo le encantaba jugar con rampas inclinadas y bolas rodantes para medir la caída de los graves. Usar rampas de poca pendiente que retardaran la caída era la única manera de hacer mediciones fiables de tiempos y espacios con los instrumentos disponibles en la época. Quizás fue ésta su mayor contribución a la física, no sólo por la ley que supo formular (el espacio recorrido es proporcional al cuadrado del tiempo transcurrido en la caída, y es independiente del peso del cuerpo), sino tal vez de modo más radical por el método de investigación empleado.

Supongamos que realizamos el siguiente experimento con la rampa en "diente de sierra" de la figura. Dejamos rodar la bola por el primer tramo hasta que llega al "muro" vertical. Entonces cogemos la bola con la mano y la subimos para que siga rodando por el segundo tramo. Supongamos que la rampa y la bola son blancas sobre una pared negra, y que la mano que mueve la bola lleva un guante también negro, de modo que no podemos verla contra el fondo negro. Podríamos incluso grabar la trayectoria con una cámara de video para analizarla a conveniencia. Nuestra observación del fenómeno puede quedar representada con las flechas punteadas de la figura. 


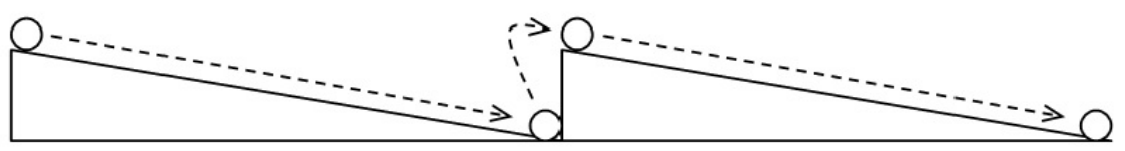

¿Qué tenemos aquí? Pues, ni más ni menos, un salto brusco, que sería el análogo a un salto brusco en la evolución humana, si es que llega a descubrirse tal salto (que como ya he comentado no parece ser el caso en el estado actual de la ciencia). La bola sigue obedientemente las leyes de la física en su primera caída, pero de repente adopta un comportamiento completamente anómalo e imprevisible: sube por el muro vertical contraviniendo la ley de la gravedad; luego, por fin, vuelve a ajustarse a la legalidad vigente en su segunda caída. Insisto, éste es el fenómeno que podemos observar, la trayectoria de la bola; otra cosa es la explicación que le demos.

En particular, es imposible explicar el salto brusco de la bola conforme a la ley de la gravedad. ¿Debemos concluir entonces que ha existido algún tipo de intervención sobrenatural que ha elevado la bola rodante hasta la segunda rampa? En cierto modo sí... puesto que la mano que eleva la bola actúa conforme a una legalidad que está por encima de la naturaleza expresada meramente en la ley de caída de los graves. Pero en sentido estricto no es en absoluto una intervención sobrenatural, puesto que los movimientos de la mano están también sometidos a las leyes de la mecánica, eso sí, en un marco de comprensión más amplio que la sola ley de la gravedad.

En definitiva, observar fenómenos que no se pueden explicar conforme a las leyes conocidas según el estado de la ciencia no permite en modo alguno postular la intervención de causas sobrenaturales. No hay un solo fenómeno físico, en tanto que fenómeno físico, que requiera la existencia de una causa meta-física. E1 104 
método científico, de por sí, no conduce a descubrir la intervención sobrenatural en la naturaleza. En palabras del libro del Prof. Arana, cuando trata de la cuestión del origen de la vida (p. 252):

Aunque los especialistas no sepan qué naipe elegir, saben muy bien con qué baraja juegan. [...] Los que investigan el origen de la vida están de acuerdo en que el problema ha de ser planteado en el terreno de la bioquímica; no tienen dudas sobre las piezas del puzzle, aunque siga escapándose la tarea de armarlo.

No hay, no puede haber, un test científico de la existencia de Dios y su intervención en la naturaleza. Esto no implica que sea imposible conocer la existencia de Dios; simplemente significa que el método científico no es el camino adecuado para alcanzar (o refutar) este conocimiento. Lo cual no tiene nada de extraño, puesto que el método científico está autolimitado a los fenómenos de la naturaleza. Esta limitación sólo es un problema para quienes consideran que el método científico es el camino privilegiado, incluso el único camino, para alcanzar el conocimiento de la realidad. El conocimiento de Dios y otras realidades trascendentes sólo puede lograrse desde un concepto más amplio de razón, que sólo será posible, en palabras de Joseph Ratzinger, "si superamos la limitación autoimpuesta de la razón a lo que se puede verificar con la experimentación, y le abrimos nuevamente toda su amplitud”3. Esta apertura de la razón a lo que trasciende lo puramente

3 J. Ratzinger. Fe, razón y universidad. Recuerdos y reflexiones. Discurso en la Universidad de Ratisbona, 12 de septiembre de 2006 (http://www.zenit.org/article-20352?l=spanish). 
fenoménico, de paso, será muy beneficiosa para muchos otros campos del conocimiento, y en primer lugar para el propio método científico ${ }^{4}$.

Arana sugiere la posibilidad de diseñar un Test de Descartes (en la línea del conocido Test de Turing) para discriminar entre seres vivos y seres inertes, y examina algunas dificultades que podría plantear este test (pp. 248-250). Lo cierto es que no podemos diseñar el test si no sabemos ya de alguna manera qué es la vida. ¿Por qué escogemos unos criterios y no otros para discriminar entre lo vivo y lo no vivo? Podemos escoger criterios discriminadores (tests) porque ya sabemos, aunque sea de modo vago e informal, qué es un ser vivo y qué es un ser no vivo. No niego la utilidad de los criterios: definirlos nos ayuda a perfilar mejor nuestro conocimiento; tan sólo digo que los criterios no están al principio del camino del conocimiento, sino más bien en la mitad, lejos también de la meta, que no puede consistir meramente en una lista de criterios, tests y procedimientos de medida.

Exactamente lo mismo ocurre con el Test de Turing, que es una prueba empírica para discriminar entre una persona y una máquina. El test fue ideado por Alan Turing, insigne fundador de las ciencias de la computación, cuyo centenario hemos celebrado en 2012. El test, que quedó magníficamente representado de forma artística en la película Blade Runner, consiste en hacer preguntas a un interlocutor hasta que quede claro si se trata de una persona o una máquina. Hoy día se ha convertido en algo cotidiano: me

4 G. Génova Fuster, M. del R. González Martín, "Cuatro problemas del método científicoexperimental que reclaman la apertura a la inteligencia meta-metódica", I Congreso Internacional de Filosofía de la Inteligencia, Madrid, 15-17 junio 2011. En M. Oriol (ed.), Inteligencia y filosofia, Madrid, Marova, 2012, pp. 661-680. 
atrevo a asegurar que cualquier lector habrá sido sometido a alguna forma particular de este test, no una sino muchas veces. En efecto, cada vez que al final de un formulario electrónico tengo que interpretar la imagen distorsionada de una palabra ${ }^{5}$, estoy siendo sometido a una prueba para verificar que soy una persona y no un proceso automatizado, y esto es algo que me ocurre ya casi a diario. El Test de Turing funciona porque sabemos de antemano que las personas lo pasan y las máquinas no, es decir, propiamente hablando no necesitamos el test para distinguir a las personas de las máquinas. El problema de esta técnica es que las máquinas que se dedican a navegar por Internet y rellenar automáticamente estos formularios mejoran continuamente, de modo que es necesario depurar una y otra vez la técnica para que siga siendo eficaz.

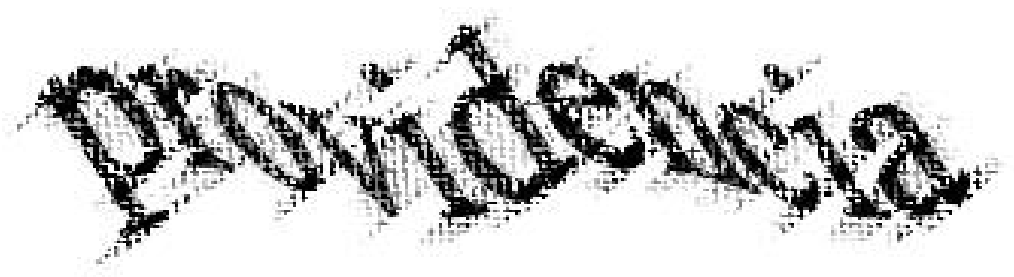

El Test del Turing es un criterio basado en el comportamiento. Pueden establecerse también criterios basados en la morfología o en el origen: es humano quien se comporta como un humano, quien tiene forma humana, quien tiene origen humano. Pero los avances de la ingeniería hacen que cual-

5 Técnicamente se denomina CAPTCHA, siglas de Completely Automated Public Turing test to tell Computers and Humans Apart: test de Turing público y automático para diferenciar entre máquinas y humanos (http://www.captcha.net/). 
quiera de estos criterios se enfrente a las mismas dificultades que el Test de Turing. La definición de ser humano como bipedo parlante era válida hace pocas décadas. Hoy ya no lo es, y cualquier otro test empírico que seamos capaces de diseñar probablemente dejará de ser válido en pocos años. Cualquier test, para ser eficaz y repetible, requiere una definición rigurosa y formal; pero es precisamente esta definición rigurosa y formal la que permite diseñar una máquina que pase el test, cuando pasar el test se convierte en un problema cerrado, entonces es planteable intentar resolverlo de forma mecánica. Así pues, no hay un criterio estático para definir lo que es una persona, y que sirva para determinar de una vez por todas, mediante un test empírico, riguroso y formal, si un ser es o no es una persona. En realidad, esto no es nada nuevo para quienes saben que persona es un concepto meta-empírico, meta-físico. Ser capaz de ver un rostro donde una cámara de video sólo graba colores en movimiento es una capacidad que va más allá de la percepción sensible de los fenómenos. Ser capaz de ver el rostro del otro es algo que está más allá de cualquier test empírico que podamos idear.

¿Tiene rostro el universo? Dicho de otra manera, ¿hay Alguien de quien podamos decir que el universo es su rostro, hay Alguien cuyo rostro podemos contemplar en el universo? A lo largo de la historia se ha intentado presentar el universo como el rostro de Dios, como una manifestación más o menos evidente de su existencia y su forma de ser. Como escribe Arana (p. 304), "los creyentes siempre vieron en el mundo vestigios confirmatorios de su fe y justo lo contrario los impíos de todas las épocas". Lo cierto es que los fenómenos naturales, de por sí, presentan una fuerte ambivalencia: "Nadie puede negar que la naturaleza en general y la naturaleza viva en particular ofrecen un espectáculo maravilloso, sorprendente, enternecedor y terrible al mismo 108 
tiempo" (p. 305). Vuelvo a la idea que ya he expuesto al principio de este comentario: la clave para llegar a contemplar el rostro de Dios en el universo no está en el análisis de los fenómenos, como si unos fenómenos apuntaran a Dios y otros más bien en dirección contraria. Aun aceptando que la compleja simplicidad del universo "claramente exbibe la marca de la elección inteligente en su entramado legal" (p. 323), para dejarse seducir por este exhibicionismo es necesario ser capaz de ver más allá de los fenómenos. Insisto, no digo que sea imposible contemplar a Dios en la naturaleza, sino sólo que los fenómenos son de por sí radicalmente insuficientes para demostrar su existencia como Creador, y menos aún como Providente. A mi modo de ver, esto que digo está en profundo acuerdo con una de las líneas de fondo del libro de Arana: "La tesis de que Dios es el Señor de la Historia no es en modo alguno refutable, ni tampoco en este sentido científica" (p. 307). No sucumbamos al cientificismo positivista: no hay un test científico de Dios, iy no tenemos por qué lamentarnos!

Gonzalo Génova

ggenova@inf.uc3m.es 
